\title{
Agôn
}

Revue des arts de la scène

$8 \mid 2019$

Matières

\section{Matières et mémoire, ou le théâtre concret de François Tanguy (Théâtre du Radeau)}

\section{Maxence Cambron}

\section{(2) OpenEdition}

Journals

Édition électronique

URL : http://journals.openedition.org/agon/6542

DOI : 10.4000 /agon. 6542

ISSN : 1961-8581

Éditeur

Association Agôn

Référence électronique

Maxence Cambron, « Matières et mémoire, ou le théâtre concret de François Tanguy (Théâtre du Radeau) », Agôn [En ligne], 8 | 2019, mis en ligne le 24 février 2020, consulté le 04 juin 2020. URL : http://journals.openedition.org/agon/6542; DOI : https://doi.org/10.4000/agon.6542

Ce document a été généré automatiquement le 4 juin 2020

Association Agôn et les auteurs des articles 


\title{
Matières et mémoire, ou le théâtre concret de François Tanguy (Théâtre du Radeau)
}

\author{
Maxence Cambron
}

1 C'est un enchevêtrement de pièces de bois aux essences diverses, d'éléments métalliques ou de plastique déclinés en tubes, en bâches, en tôles. Le tout agencé, bricolé, pour donner forme à des tables, des cadres, des châssis à la stabilité précaire. Dans les anfractuosités de ce paysage de décharge passent des corps dans des frottements de laine, des bruissements de satin, des cliquetis d'armures et autres surgissements de tulles, de plumes ou de fourrures. Un déferlement de matières défraîchies, mises en mouvement dans des atmosphères de lumières et de sons participant d'une forte texturation de la scène.

2 Cette symphonie matérielle aux allures de rhapsodie est celle qui, la représentation durant, sollicite et active en continu le regard du spectateur du Théâtre du Radeau. Des espaces conçus et construits par François Tanguy, metteur en scène de la compagnie depuis $1982^{1}$, se dégage en effet une puissance poétique tout à fait singulière fondée sur une certaine qualité de présence accordée aux éléments scénographiques, objets et costumes accumulés et agencés sur scène ${ }^{2}$.

3 S'inscrivant dans le lignage kantorien d'une approche plastique et concrète de l'espace scénique, cette relation à la matière s'ancre dans le souci résolument postdramatique d'émanciper l'art du théâtre de ses traditionnelles assignations mimétiques, dramatiques et textocentriques au profit d'une conception formée sur un primat du présent et de la perception. Ce privilège accordé aux sens, et plus précisément à l'acte du regard, est particulièrement prégnant dans la démarche de François Tanguy, qui n'a de cesse d'en référer à l'étymologie du mot "théâtre " (le lieu d'où l'on voit) pour asseoir son propre geste théâtral. Cette prédominance affirmée du voir est d'importance car elle détermine l'usage et le fonctionnement de la dimension matérielle de son travail : matières et matériaux, utilisés dans des constructions (pour l'élaboration des agrès qui constituent l'essentiel de la scénographie) ou transformés 
(entrant dans la composition des objets ou des costumes) sont ainsi disposés dans l'espace selon un principe d'immanence qui offre les matières au regard du spectateur d'abord et avant tout pour ce qu'elles sont, telles qu'elles sont, avant d'en faire des supports de signifiance ou de symbolisation; ce qui pousse François Tanguy à revendiquer que, dans son théâtre, «ce que nous voyons n'est pas le code de ce que nous ne voyons pas, ce qui est à voir est très exactement ce que nous voyons, ce que nous pouvons voir ${ }^{3}$ ".

4 S'il se concentre ainsi sur une présence autoréférentielle de la matière, ce principe d'immanence n'en laisse pas moins la place à une riche complexité de cette présence même, et ce, sur deux plans. D'abord, parce qu'en s'appuyant sur une pratique de la récupération et en manifestant un intérêt pour les matières diversement "datées " (traces d'usure, référence à un style, une époque...), le travail de François Tanguy établit un lien très direct entre la matière et le temps - la matière se voyant ainsi chargée d'une double mémoire: celle qui témoigne d'un passé humain et la sienne propre. Ensuite, parce qu'en refusant d'envisager son geste scénographique comme une installation figée et potentiellement autonome, mais au contraire comme un composant essentiel du jeu théâtral, voire comme l'endroit d'une relation spécifique entre les acteurs-actrices et la matière, le metteur en scène développe, avec sa compagnie, un art de l'acteur tout à fait singulier basé sur un nouveau partage des tâches entre l'humain et la matière.

5 Ainsi, par ce souci de libérer les matières de toutes fonctions ancillaires (utilitaires, illustratives ou figuratives) et de mettre en jeu leurs vibrations propres, tout comme par la mise en œuvre d'un partenariat théâtral entre ces matières et les acteurs, il apparaît que la démarche de François Tanguy pourrait être rapprochée des idées des nouveaux matérialismes ${ }^{4}$. Bien que ce lien ne soit pas revendiqué par l'artiste, notre étude proposera de creuser les correspondances nombreuses et opérantes qui se font jour entre sa pratique et ce courant de pensée. En nous focalisant sur le statut spécifique que François Tanguy confère aux matières, tant pour leur participation à la conception visuelle du spectacle que pour leur fonction dramaturgique singulière, nous interrogerons la façon dont elles s'insèrent dans la poétique théâtrale du metteur en scène et la part active qu'elles prennent dans son écriture scénique pour dire la complexité du temps - entre matières et mémoires. 
Figure 1 - L'espace de Soubresaut (2016)

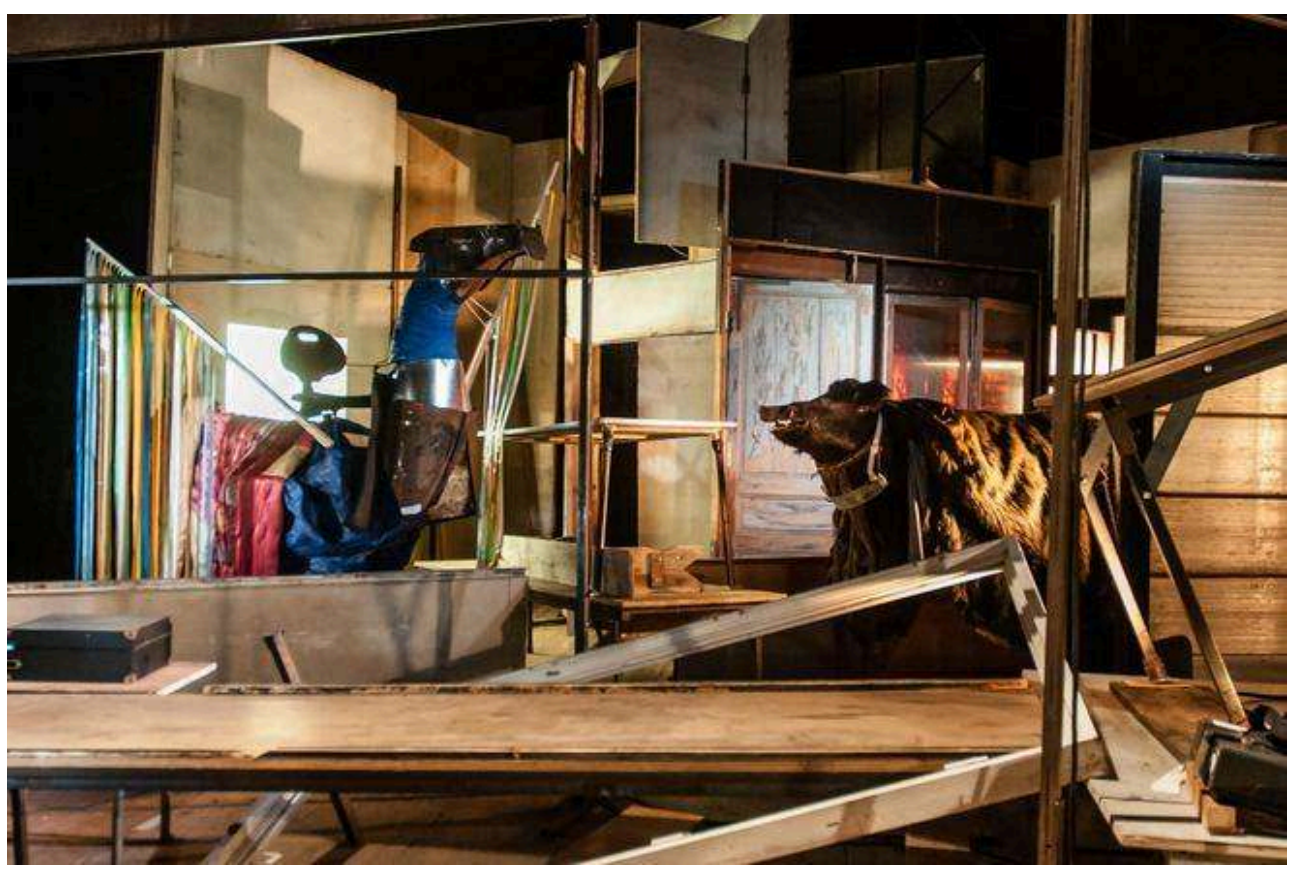

(Crédit photo : Karine Pierre)

\section{Matérielle rhapsodie}

6 Ô combien reconnaissable dans le paysage théâtral européen, l'identité visuelle du Théâtre du Radeau se caractérise par une saturation matérielle de l'espace scénique. Bois massifs, bruts ou traités, ou encore plaqués, contreplaqués, agglomérés ou mélaminés ; fer, acier, aluminium et autres métaux ; plastiques divers transformés en plaques lisses ou ondulées, en bâches opaques ou transparentes, en balatums ou en toiles cirées; cartons et papiers ; fibres textiles ; toiles d'ameublement; verres et miroirs patinés; fleurs et branchages (souvent séchés après avoir été glanés dans la nature sarthoise), plumes, cornes et fourrures (généralement employées comme pièces de costume, mais parfois toujours en place sur l'animal - cependant empaillé) : même compressé, l'impressionnant inventaire des matières requises par François Tanguy dans ses scénographies frappe également par son hétéroclisme.

7 Si aucun n'est particulièrement privilégié, on remarquera néanmoins une prédominance des matériaux pauvres et courants issus de procédés industriels et chimiques $^{5}$, tels que le fer (pour les structures des tables, cadres et châssis), les matières transformées à partir du bois (planches de contreplaqué, mobilier en Formica...) ou les différentes matières plastiques (polyéthylène, polypropylène, PVC...) des nombreuses bâches et tôles qui emplissent l'espace. Artefacts reliés au monde moderne et à la vie quotidienne, ces " matériaux vils » selon l'expression de Florence de Mèredieu ${ }^{6}$ sont ainsi rassemblés et travaillés par le metteur en scène depuis près de trois décennies au gré d'une pratique de la récupération, du réemploi et du bricolage au travers de laquelle se manifeste une évidente influence kantorienne. 
Figure 2 - L'espace (hors représentation) d'Item (2019)

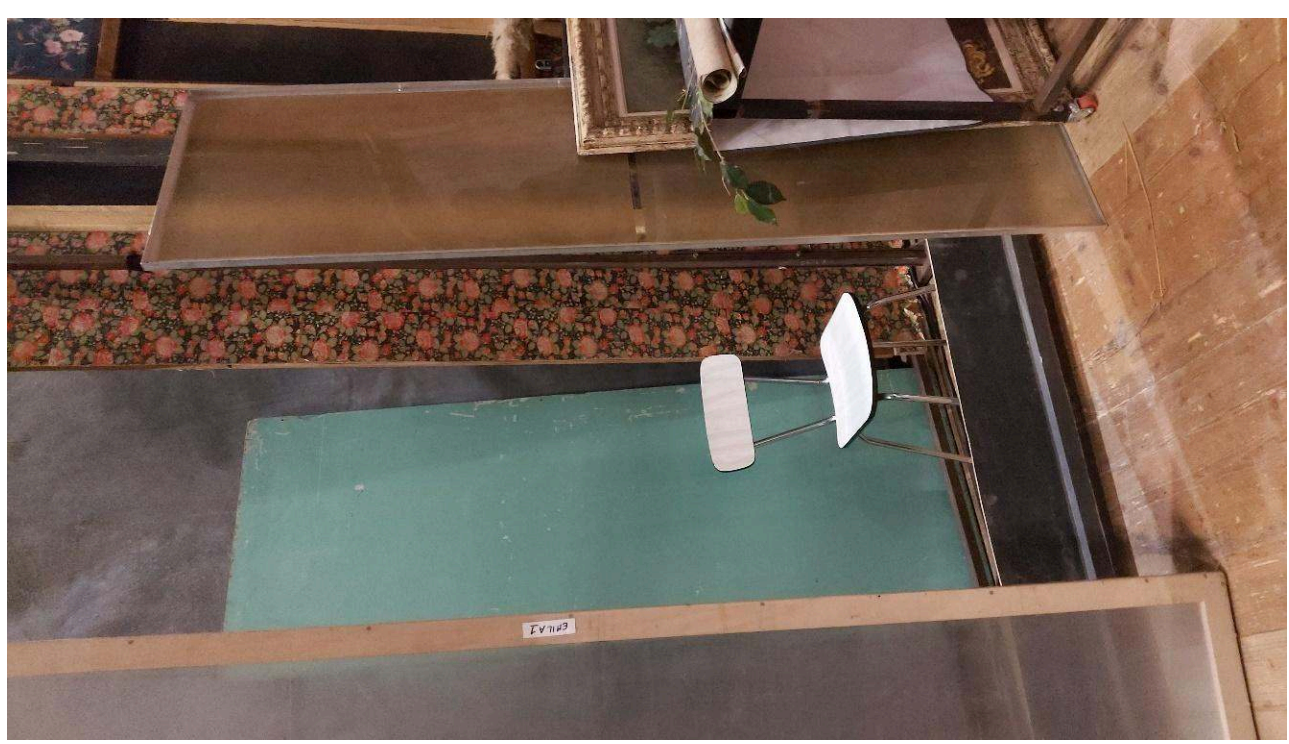

(Crédit photo : Maxence Cambron)

8 Contemporain de démarches artistiques qui, dans le domaine des arts plastiques (l'arte povera, Beuys, Arman, Dubuffet, Spoerri...), ont entrepris la valorisation de «matériaux innommables $»^{7}$ (tels les matières organiques ou les déchets, dont la présence était jusqu'alors impensable dans le champ artistique dominé par les canons de l'art académique bourgeois), Tadeusz Kantor - lui-même plasticien - transposa dans le domaine théâtral cette même recherche d'indépendance vis-à-vis de la forme et des conventions. Quête fondamentale de l'artiste: affranchir le théâtre du système mimétique en cherchant «l'expression de la réalité par la réalité elle-même - non par l'imitation de celle-ci ${ }^{8} »$. Dès les années 1940 , il intégra ainsi à ses premières réalisations des objets et matériaux de récupération - non pas à des fins de figuration historique ou réaliste, mais avec le désir de faire entrer sur scène ce qu'il nomma la " réalité du rang le plus bas ». Bois vermoulus, métaux oxydés, tissus élimés et autres matières réchappées de la benne commencèrent d'occuper les espaces kantoriens pour ne plus les quitter. Pour Kantor, ces matériaux, saisis "entre éternité et poubelle ", autrement dit « arrachés à la vie même ${ }^{10}$ » mais sauvés de la destruction, voire rédimés par le geste créateur, devinrent ainsi les catalyseurs d'un "concrétisme ${ }^{11}$ qui, sans confondre le réel et le théâtre, inscrivit ce dernier de plain-pied dans la sphère du réel. L'artiste se fit donc récupérateur, circulant dans les rues, les greniers et les décharges pour collecter, au hasard des rencontres, les objets, les matières et les matériaux qui allaient nourrir son imaginaire et peut-être intégrer sa fabrique théâtrale. Ces principes ainsi formulés par le maître polonais font fortement écho à la pratique de François Tanguy, chez qui se met également en œuvre une esthétique - voire, par certains aspects, une éthique doublée d'une économie ${ }^{12}$ - de la récupération et du collectage de matières et d'objets "pauvres ${ }^{13}$ ", défraîchis ou usés, qui n'est pas sans rappeler le monde oublié des chiffonniers récemment remis au jour par Antoine Compagnon ${ }^{14}$.

Depuis le début des années 1990, le metteur en scène constitue en effet dans les locaux du Radeau, au Mans, une véritable collection de matières, matériaux, objets et costumes. Glanés (ou « chiffonnés ») dans les bric-à-brac Emmaüs, les vide-greniers, les 
déstockages et autres fins de chantiers des environs, ces éléments composent une sorte de matériauthèque du reste et du déchet dans laquelle il va puiser afin de donner forme, par associations, constructions, reconstructions et restructurations successives, à ces cavernes encombrées d'artefacts.

Cette réappropriation des matériaux est un geste fort de l'approche plastique et visuelle de François Tanguy, qui s'implique personnellement dans la construction de ces éléments. Celui-ci n'en fait d'ailleurs pas son privilège exclusif puisqu'une partie de la compagnie (techniciennes, acteurs ou actrices, aides amicales de passage) prend souvent part à l'élaboration concrète de la scénographie - c'est-à-dire sa conception intellectuelle mais surtout manuelle (le Radeau ne sous-traite ni l'une ni l'autre de ces deux étapes essentielles au travail de fondation d'une nouvelle création) ${ }^{15}$. Car au Radeau, les répétitions débutent à l'atelier, là où scieuses, cloueuses et autres postes à souder précèdent la boîte à outils dramaturgique ${ }^{16}$. Dans ces prémices, desquelles débouchera l'élan d'un nouveau spectacle, l'ensemble (metteur en scène, acteurs et actrices, amitiés de passage) rhapsode l'espace, c'est-à-dire compose et recompose les structures - tables, cadres, châssis, écrans et autres panneaux - sur lesquelles s'appuie la grammaire scénographique du Radeau.

Pour tous les participantee's à la création, s'immerger dans les matières constitue alors un préalable essentiel à l'approche à proprement parler théâtrale car les rencontrer dans cette antichambre du plateau qu'est l'atelier de construction des "décors ", les manipuler, les transformer, les bricoler est une façon de commencer à jouer avec elles (ce qui prendra tout son sens dans le troisième temps de notre réflexion) en apprenant à connaître et reconnaître leur manière de prendre place dans l'espace, celle de réagir au déplacement, à la lumière, ou encore d'expérimenter leur degré de résistance au poids d'un corps humain (s'il s'agit de s'y jucher). En d'autres termes, se montrer attentif à une potentielle vitalité des matières et entamer avec elles une relation sur laquelle repose une part essentielle du travail théâtral. 
Figure 3 - Les Cantates (2001). Frode Bjørnstad / Fosco Corliano

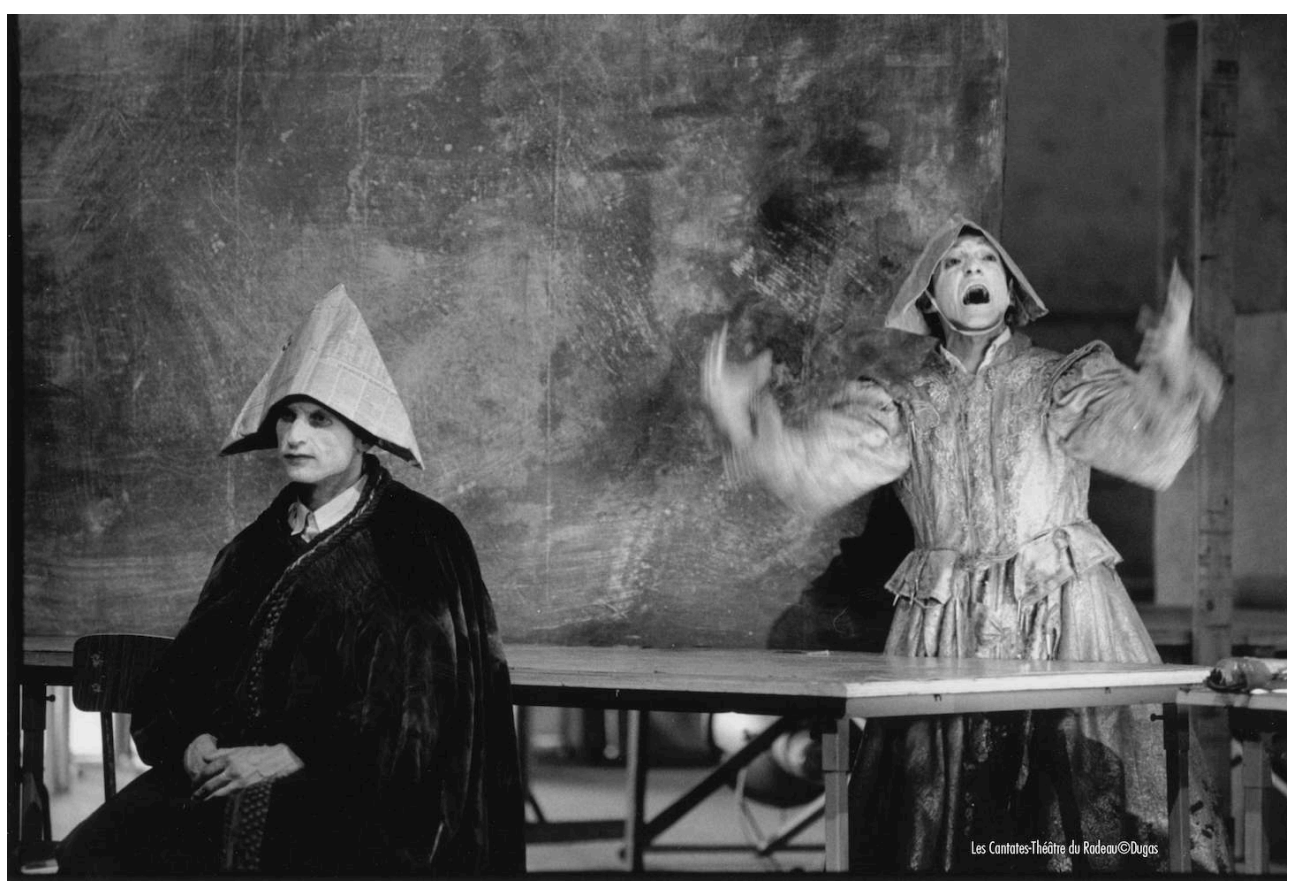

(Crédit photo : Dugas)

\section{Matières vibrantes, matières mémorantes}

\section{Un autre regard sur les matières}

La critique (universitaire comme journalistique) a souvent eu recours à des comparaisons pour décrire les scénographies du Radeau. D'aucun y voit l'atelier d'un peintre dont toutes les toiles seraient retournées, ou encore la réserve empoussiérée d'un théâtre à l'abandon. Ces images, loin d'être inopérantes, ont néanmoins un défaut : elles déportent l'attention. Selon elles, panneaux, cadres et châssis conçus par le metteur en scène ne seraient que des supports vierges - des objets passifs, en attente, et sur lesquels il n'y aurait rien à voir. Or, précisément, rien d'autre n'est à voir qu'euxmêmes, et cela est déjà remarquable.

Dans son ouvrage Faire. Anthropologie, archéologie, art et architecture ${ }^{17}$, Tim Ingold rappelle que notre expérience moderne du monde (occidental), influencée par le positivisme et l'esprit scientifique, a perdu en épaisseur en réduisant notre compréhension de ce monde à une connaissance rationnelle de ses constituants ${ }^{18}$. L'anthropologue britannique plaide alors pour un retour à un mode de perception inspiré des anciens savoirs alchimiques et, partant, à une forme de réenchantement du monde. Reprenant à son compte la distinction opérée dans les années 1960 par David Pye entre " propriétés » et " qualités » de la matière - les premières étant « objectives et scientifiquement mesurables ${ }^{19}$ les secondes, « subjectives, [étant] des idées que les gens se font dans leur tête et qu'ils projettent sur le matériau en question »-, Tim Ingold en vient ainsi à défendre une lecture plus complexe de notre rapport à la matière et, plus généralement, une sorte de relativisation générale des liens qui peuvent unir les humains (dans son propos, les artisans) aux matières. 
Chacun de leurs gestes techniques, écrit-il, est une question, à laquelle le matériau répond selon ses dispositions. En suivant leurs matériaux, ce n'est pas tant que les artisans « interagissent ", mais plutôt qu'ils " correspondent » avec eux. Et donc, fabriquer est un processus de correspondance, et non l'imposition d'une forme préconçue sur une substance matérielle brute, mais la prolongation ou la présentation de potentiels immanents dans un monde de devenir. Dans le monde phénoménal, chaque matériau est un devenir, un chemin ou une trajectoire à travers un labyrinthe de trajectoires.

13 Selon un même tropisme alchimique qui témoigne d'une attention accrue portée à leurs singularités et d'une volonté d'entrer avec elles dans un «processus de correspondance ", tout l'art de François Tanguy nous semble pareillement consister à laisser jouer les matières en se mettant à l'écoute de leurs propriétés (forme, taille, couleurs, densité, transparence ou opacité...) en évitant de les asservir à un projet esthétique préétabli.

Sur ce point, l'ascendance kantorienne paraît de nouveau avoir joué un rôle majeur. En effet, une page d'un manifeste de la première moitié des années $1970^{20}$ laisse entendre que l'intérêt du maître polonais se portait également sur les qualités propres de la matière. Revenant sur sa quête d'un "théâtre informel » au début des années 1960, il revendiquait alors un «tournant important dans le développement du Théâtre Cricot 2 » : la découverte d'une poussée vitale d'une matière "palpitante ", " affranchie des lois de la construction [...] et devenue pays d'aventure pour l'art et la conscience humaine ". Comment, à travers ces mots, ne pas tisser de lien avec ceux de la philosophe américaine Jane Bennett qui, avec Vibrant matter ${ }^{21}$, signa l'un des ouvrages phares des pensées néo-matérialistes?

une perspective trans-humaniste et surtout « désanthropocentriste » cherchant à battre en brèche les représentations de l'humain " maître et possesseur de la nature ", la philosophe postule «une agentivité des forces non-humaines (opérant dans la nature, dans le corps humain et dans les artefacts humains) [qui tente] de contrer le réflexe narcissique du langage et de la pensée humaine ${ }^{22}$ ». En s'appuyant sur la phénoménologie de Merleau-Ponty ${ }^{23}$, la théorie des affects de Spinoza ${ }^{24}$, celle des actants de Bruno Latour ${ }^{25}$, ou encore celle de la " matière-flux » de Deleuze-Guattari ${ }^{26}$, la pensée néo-matérialiste de Jane Bennett consiste ainsi à «donner la parole à une vitalité intrinsèque [aux] matières ${ }^{27} »-$ une " pâte de vie $»^{28}$ pour emprunter les mots de Kantor dans le même texte que celui précédemment cité - dont l'une des résultantes serait la prise d'indépendance de celles-ci «vis-à-vis des mots, des images et des sentiments [qu'elles] provoquent en nous ${ }^{29} »$.

Cherchant à ne pas remplacer un dualisme par un autre, la thèse de Jane Bennett pose en outre le principe d'une " continuité » ${ }^{30}$ entre les êtres et les choses, l'humain et la matière (qu'elle soit organique ou non). Selon elle, il serait donc possible d'envisager un circuit affectif multilatéral dans lequel l'humain et la matérialité non-humaine s'affecteraient mutuellement tout comme les différentes sphères de ladite matérialité s'entre-affecteraient elles-mêmes; ce qui rejoint en partie le principe du « processus de correspondance » évoqué par Tim Ingold quelques années plus tard, voire même celui de la «trajection » proposé par Augustin Berque dans sa mésologie depuis la fin des années $1980^{31}$.

Selon ce dernier, la trajection - dont l'exemple type est "l'invention du paysage ${ }^{32}-$ consiste à faire exister un être ou une chose dans le milieu donné duquel il participe (et qui peut être différent selon un autre point de vue mésologique : ainsi, un même brin 
d'herbe participe potentiellement de plusieurs milieux, par exemple celui d'une vache, d'un insecte ou d'un humain, dans lesquels il n'occupe pas la même fonction) ${ }^{33}$. Posant un équilibre entre objectivité et subjectivité, cette trajection revient donc à reconnaître autant l'existence autonome de ce qui participe de ce milieu que la construction qui lui donne sens et, plus généralement, l'organise selon différentes intentions (la faim, la recherche de hauteur, le plaisir esthétique pour reprendre les cas de figure autour du brin d'herbe).

Cette trajection est donc affaire de relation mais également de façonnage d'un milieu par un point de vue (humain ou animal). Penser le geste scénographique de François Tanguy en ces termes nous invite donc à faire la part entre l'intuition qui consiste à attribuer à la matière une autonomie " désanthropisée » et la position reconnaissant qu'il s'agit là encore d'une projection humaine portée sur les choses, y compris lorsqu'il s'agit de vouloir se déprendre du contrôle humain sur le monde.

Repérer la vibrance des matières convoquées au plateau, jouer avec / faire jouer leurs propriétés physiques en relation avec l'espace, la lumière ou les autres matières alentour, tel semble donc être le projet - humble et difficile ${ }^{34}$ - de François Tanguy. À cet égard, un panneau de contreplaqué n'en vaut pas un autre, deux bâches, même conçues dans une matière semblable, ne s'inscriront pas de la même manière dans le «milieu » formé par toutes les autres choses et matières assemblées construit par l'artiste. Sur telle pièce de bois choisie par le metteur en scène apparaîtront des veines ou un nœud que telle autre n'aura pas. La densité de cette tôle en PVC n'absorbera pas la lumière comme cette autre, qui elle-même sera parcourue de stries et au travers de la transparence desquelles le regard du spectateur verra un corps ou le reste de l'espace se perdre dans un brouillard matériel.

19 Kaléidoscopique et vertigineux, ce jeu des matières dénote donc une tentative de déprise de la posture démiurgique du geste créateur. Bien loin de l'image d'un dompteur imposant aux choses sa volonté, on peut saisir la pratique de François Tanguy comme une déterritorialisation, un déport permanent des habitudes de l'artiste lui-même mais également du spectateur, sans cesse troublé dans sa perception/ réception par cette anti-hylémorphisme en acte. Voilà notamment pourquoi François Tanguy refuse catégoriquement que son travail soit placé sous le vocable de "théâtre d'images $»^{35}$ : l'espace scénique n'est pas traité de manière esthétisante, ni même picturale, mais surtout rien ne s'y fige jamais, tout y est en mouvement et remuement, la matière même est mouvement. 
Figure 4 - Coda (2004). La silhouette de Boris Sirdey

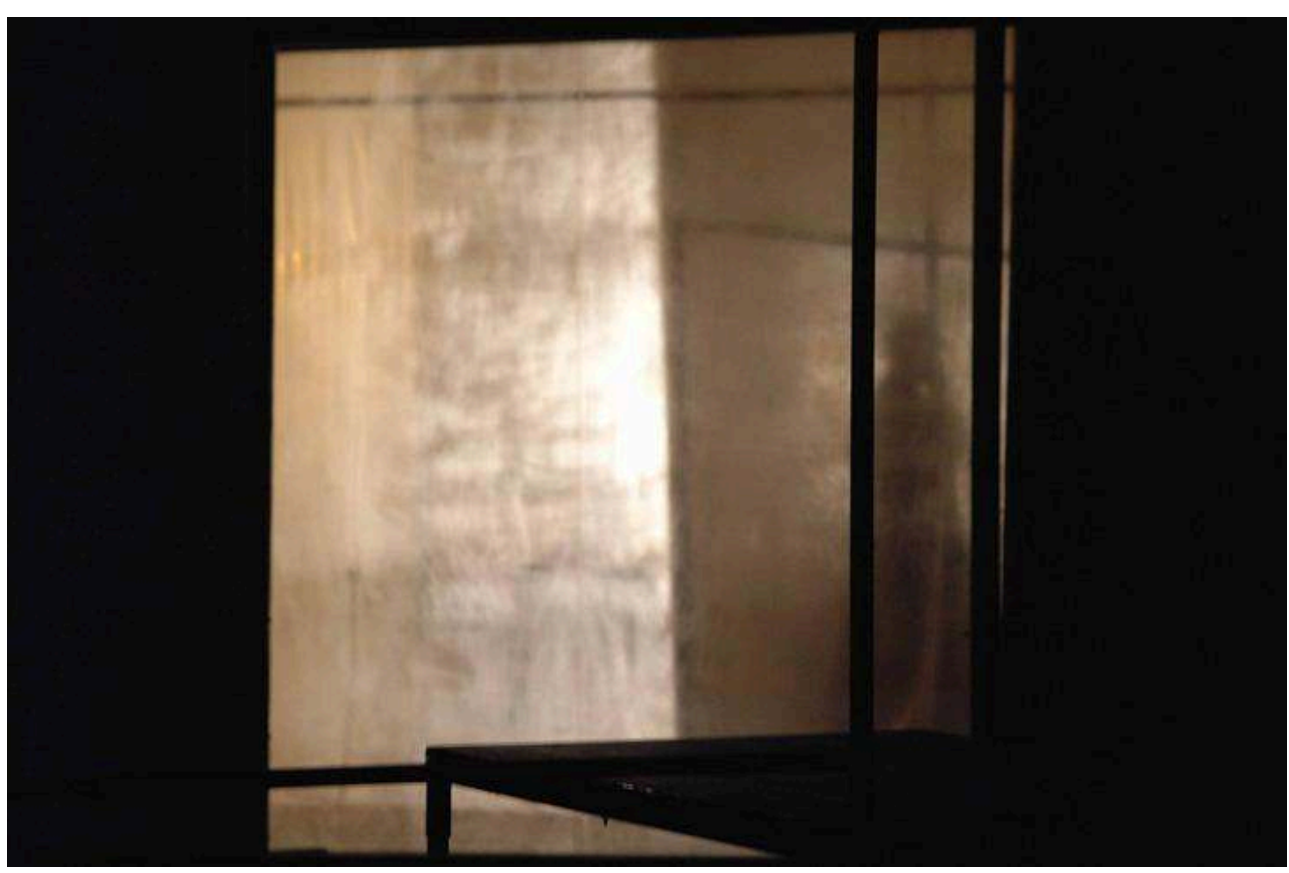

(Crédit photo : Didier Grappe)

\section{Matière-mémoire}

Si, comme nous venons de le voir, la singularité du geste scénographique du Radeau se situe à l'endroit d'une intensification de la présence matérielle (la matière faisant, en un sens, corps avec elle-même : le bois produit du bois, le fer du fer et ainsi de suite), il en est une autre, particulièrement opérante d'un point de vue dramaturgique, qui invite à envisager la matière non plus comme "pure présence " mais, au contraire, comme étant capable d'incarner le temps et de rendre palpable (à travers la variété de ses textures, de ses formes ou de ses densités) une présence concrète du temps au cœur même de l'inanimé.

La pratique de la récupération que nous évoquions précédemment est étroitement liée à cette infiltration du temps dans la matière : usées, abîmées, "meurtries " $^{36}$ selon la belle expression de François Dagognet, les matières de seconde main, comme l'écrit encore Florence de Mèredieu, "résume[nt] à [elles] seul[es] tout un passé et cristallise[nt] un savoir-faire ${ }^{37} »$. Vestiges tangibles du passage des hommes, les objets gardent l'empreinte de cette inscription physique et concrète dans le temps: ils sont les traces d'une culture matérielle autant que matérialisée et témoignent aussi bien de leur usage que de leurs usagers ${ }^{38}$.

Dans la démarche de François Tanguy, la matière se fait ainsi «mémorante " (pour reprendre un terme souvent employé par le metteur en scène ${ }^{39}$ ), lieu et occasion de rémanences pour la compagnie comme pour les spectateurs. Sur une dizaine de créations depuis la fin des années 1980, il semble en effet que ce soient les mêmes choses qui peuplent les espaces du Radeau. En découvrant une nouvelle création, on est toujours frappé par la sensation d'avoir laissé l'agencement de cadres, de châssis et de panneaux tel qu'il s'était figé à la fin du précédent. On y reconnaît les mêmes longues tables de collectivités, les chaises en formica, les grands châssis bâchés ou encore les 
panneaux tapissés des mêmes papiers-peints à motifs floraux. L'ensemble créant un fort sentiment de familiarité avec ces matières qui semblent avoir leur place dans la compagnie autant que les acteurs ${ }^{40}$.

Ce sentiment de familiarité avec les choses est renforcé par une étonnante méthode utilisée par le Radeau depuis plusieurs années pour les identifier toutes avec précision et particulièrement pour l'installation ou le rangement de la scénographie lors des tournées ou pour son stockage entre deux phases de création. Chaque élément, du châssis au moindre bâton, dès l'instant où il entre dans la configuration scénographique, se voit étiqueté et surtout nommé. Ces noms répondent à une classification précise qui présente avant tout un intérêt pratique sans pour autant délaisser le champ du poétique : les tables, par exemple, se voient attribuer des noms d'îles - « Frioul », "Groix », « Ouessant », « D'Arz »... -, ce qui achève symboliquement de faire de leur assemblage un archipel mouvant. D'autres agrès (en l'occurrence les cadres) portent les noms des personnages issus de la littérature mondiale. Il est surtout étonnant de constater que tous les panneaux et châssis portent les prénoms de grands écrivains ou musiciens dont la scène fait revivre les voix, ou d'artistes amis qui ont marqué l'histoire de la compagnie. De la sorte, au premier plan de la scénographie de Onzième (2011) se trouve un panneau baptisé « Klaus » en hommage à Klaus Michael Grüber $^{41}$.

Figures 5 et 6 : Exemples de « nomination » des agrès dans Item (2019)

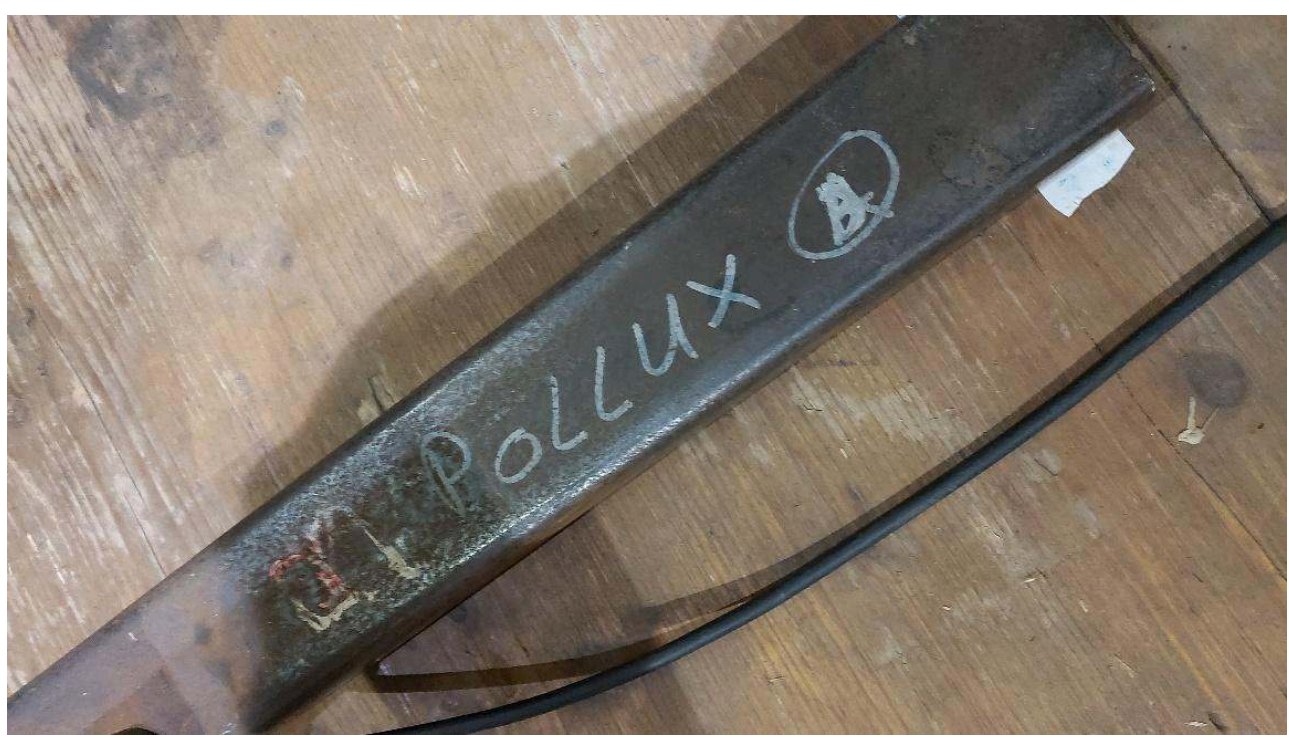



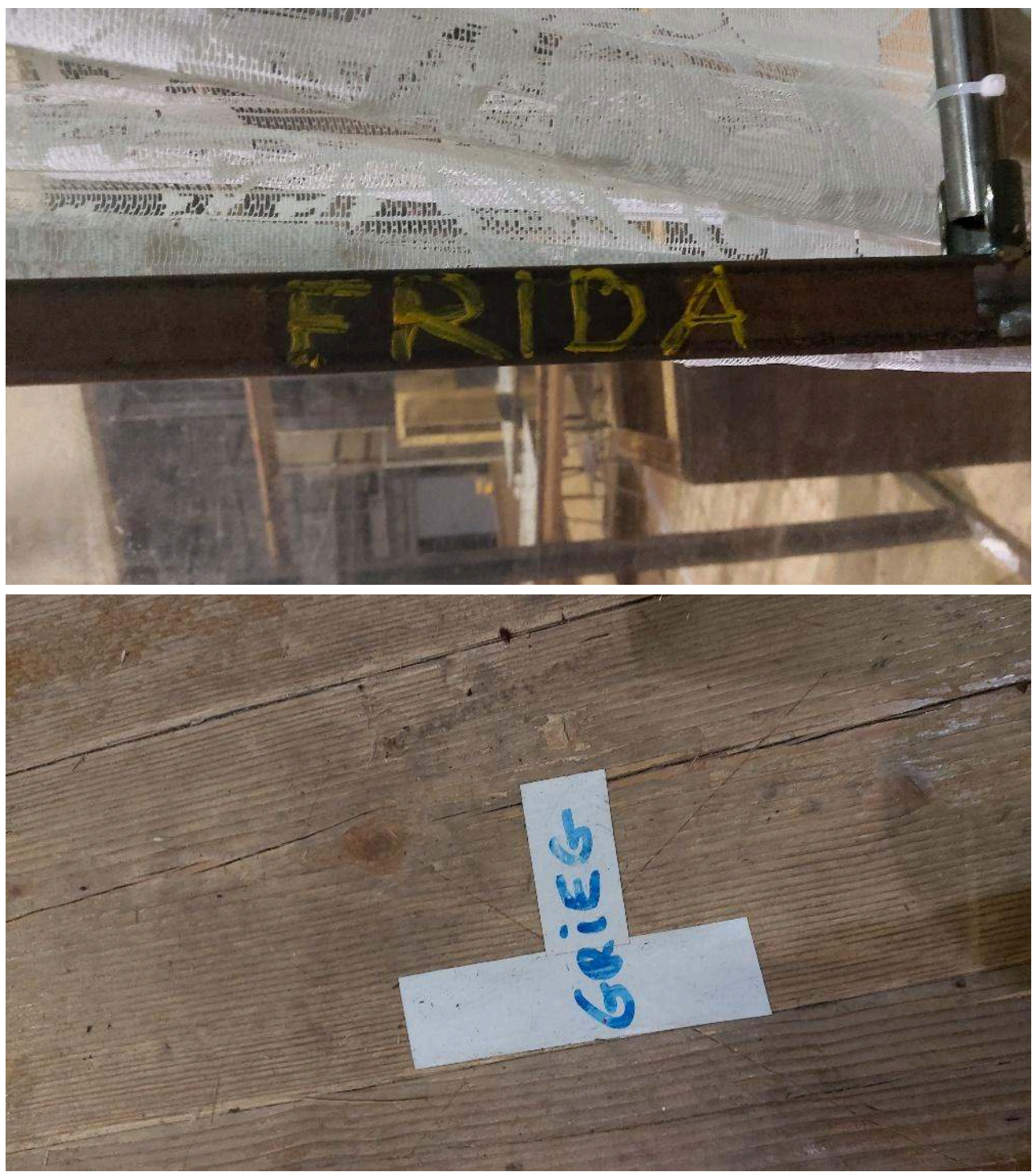

(Crédits photos : Maxence Cambron)

24 Sur les croquis d'implantation des spectacles, sous chacun des traits représentant une table ou un cadre afin de garder en mémoire l'agencement des différents agrès selon les séquences, la multitude de ces noms dessine une cartographie spectrale. Les choses s'apparentent ainsi à des réincarnations matérielles des amitiés de la compagnie, une présence en même temps qu'une mémoire, ce qui renforce d'autant plus le sentiment d'un plateau littéralement hanté, habité par de multiples esprits à la fois inquiets et bienveillants.

Par cette simple mais belle idée de «nomination » des choses - qui peut conduire, en répétitions, à entendre un acteur interroger un partenaire pour savoir si c'est bien à lui de déplacer «Emil» [Cioran] (en parlant d'un écran de contreplaqué sommairement peint en blanc, fixé sur une structure de barres d'acier à l'équilibre précaire dont on peut imaginer qu'elle a pris part à de nombreuses autres créations) avant de s'exécuter avec une certaine déférence - les choses se voient ainsi traversées par des forces mémorantes qui font d'elles des seuils vers d'autres dimensions du réel - un réel non plus simplement pragmatique mais chargé d'imaginaire, d'interprétation ou encore de mémoire. 

mémoriel de la matière. Il occulte la mémoire propre de la matière qu'à la suite de Jane Bennett nous pourrions intégrer au "pouvoir des choses " (the force of things). Car si la matière est parcourue par un « élan vital » (Bergson), c'est aussi qu'elle est régie par sa propre temporalité. La matière n'échappe pas au temps et à ses effets. Une matière qui vit est donc également une matière qui vieillit. Si notre système perceptif, convaincu de l'inertie des matières non-organiques, n'est pas apte à détecter ce changement continuel de la matière, c'est que nous avons perdu la sensibilité à la polyrythmicité de nos milieux naturels et non-naturels ${ }^{42}$. Comme l'écrit encore Jane Bennett dans un commentaire sur la pensée de la matière dans Mille plateaux de Deleuze-Guattari, les choses avec lesquelles nous cohabitons sont

des matériaux mobiles et hétérogènes dont le débit de changement interne et la vitesse extérieure sont lents comparés à la durée et à la vélocité des corps humains qui les perçoivent. Les «objets» sont des matérialités mobiles qui vieillissent plus lentement que nous, qui s'altèrent à une échelle inférieure au seuil de la perception humaine ${ }^{43}$.

Sauf cas rare, nous ne pouvons pas voir «à l'œil nu » la matière se corroder. Nous pouvons constater sa dégradation mais nous ne pouvons assister concrètement à son développement - ce que la technique du time-lapse nous permet de voir. Et pourtant cette matière n'est pas hors du temps. Nous partageons le même temps qu'elle mais selon d'autres rythmicités singulières qui demandent un effort pour les percevoir ou simplement les imaginer.

Ainsi les matières du Théâtre du Radeau sont-elles des surfaces d'enregistrement d'une mémoire externe - qu'elles symbolisent plus qu'elles ne mémorisent concrètement autant que des choses elles-mêmes mémorantes. Chargées d'une histoire intuitivement perceptible dans les traces multiples qui témoignent d'une existence antérieure au théâtre, ou encore d'une force d'évocation méta-mémorielle qui historicise leur place dans la chronologie des créations de la compagnie, la présence des matières n'est donc pas réduite à leur seule existence inerte.

Figure 7 : Item (2019). Laurence Chable

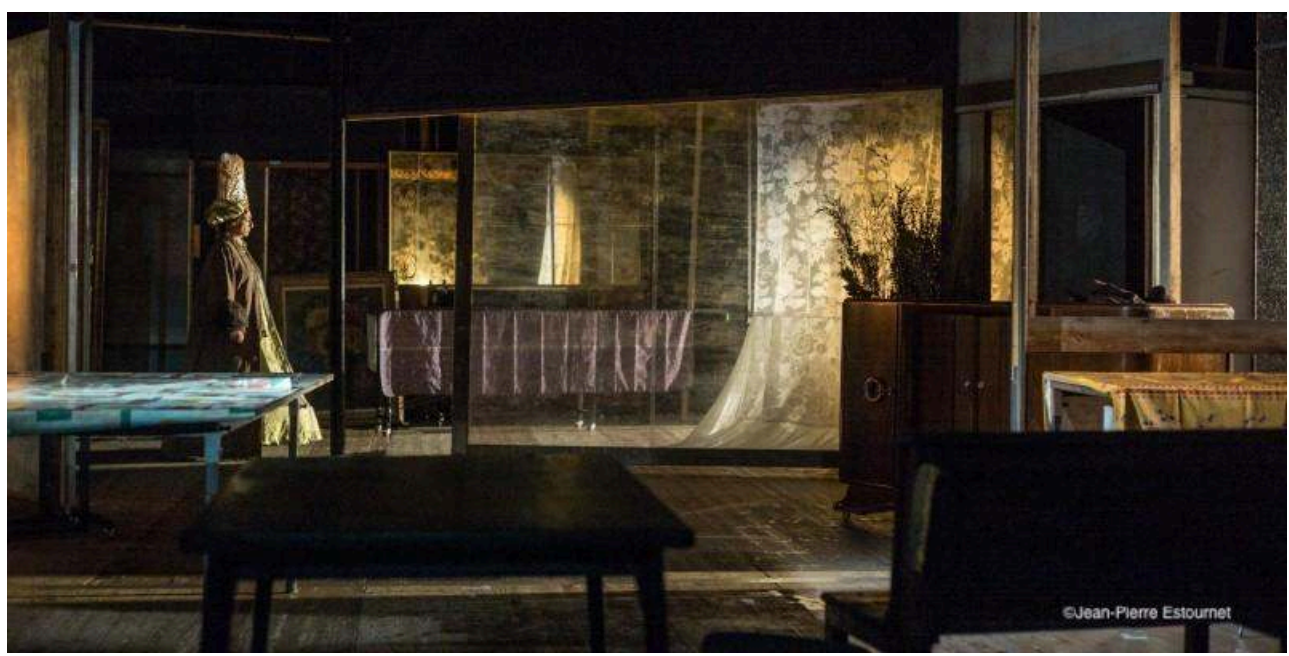

(Crédit photo : Jean-Pierre Estournet) 


\title{
Le devenir actrice de la matière
}

29 Reconnaître les pulsations vibrantes et mémorantes de la matière, lui faire prendre en charge une partie du jeu n'équivaut cependant pas à une survalorisation de son agentivité et/ou de sa présence au détriment de celle des acteurs. Rétif à la qualification de théâtre "plastique ${ }^{44}$ ", François Tanguy ne conçoit pas ses scénographies comme des dispositifs autonomes au sein desquels l'acte théâtral ne ferait que prendre place. Dans la démarche du Radeau, la présence des acteurs fait au contraire jeu égal avec la matière - ce qui nous permet de revenir aux notions de "continuité", de "processus de correspondance» et de «trajection" que nous évoquions plus haut.

30 Comme l'affirme en effet Laurence Chable, actrice fondatrice de la compagnie,

les différents éléments du plateau (tables, chaises, cadres, objets) travaillent avec toi et tu travailles avec, mais pour autant, ce n'est pas ça, le jeu. Le cadre existe pour lui-même, et si tu t'appuies, tu ne dois pas montrer que tu t'appuies sur ce cadre. Il travaille avec toi, il prend en charge quelque chose. Tu n'as pas à lui voler la place en montrant que tu t'y appuies. Tu n'écrases pas. On joue ensemble ${ }^{45}$.

31 Ces phrases, éminemment éclairantes pour notre propos, nous font parfaitement entendre à quel point le travail des acteurs du Radeau consiste non seulement à détecter les appuis de jeu dont l'espace peut être la source, mais également à nouer une relation de réciprocité avec le milieu sensible que forment ces rassemblements de choses et de matières. "Jouer » sur la scène du Radeau consisterait donc à mettre en œuvre une recherche des correspondances (corporelles, vocales, rythmiques...) entre un corps et une matière qui rallierait la finalité toute " postdramatique » visant à émanciper la scène du traditionnel surplomb qu'y occupe le texte, en laissant "agir [un] écart critique ouver ${ }^{46}$ » qui opère comme un intercesseur - pour reprendre un terme employé par Laurence Chable - des potentialités théâtrales des matières convoquées au plateau. Sur cette même question, le témoignage de l'acteur Dominique Bénard est éloquent :

\begin{abstract}
L'acteur du Théâtre du Radeau est d'abord un ouvrier de plateau. L'espace-temps du spectacle est un ensemble de plis et de déplis fait par ces acteurs-ouvriers. [...] Je me souviens m'être dit que ce n'était pas moi qui parlais, mais la planche, et que si elle parlait, c'était parce qu'il y avait un rayon de lumière qui l'éclairait. Le corps de l'acteur du Radeau est ainsi d'abord le corps d'un ouvrier-artisan. Il déplace des objets et des panneaux sur la scène qui dans ces déplacements acquièrent une tension. Celle-ci agit éventuellement sur ce qu'il va dire. Ses mouvements, ce ne sont pas les siens, ce sont des mouvements de l'espace et du temps qui s'actualisent dans son corps, dans sa voix ${ }^{47}$.
\end{abstract}

32 Cette mise en "tension" entre corps et matières est également prégnante dans la relation aux tissus qui composent les costumes. Ceux-ci, comme tout le reste des éléments matériels du spectacle, sont pour une très large part d'entre eux assemblés à partir de vêtements de récupération. La qualité de la matière, la coupe du vêtement, ses défauts, l'époque à laquelle il fait référence, sont autant de points d'entrée pour le jeu.

Un vêtement, particulièrement lorsqu'il est de seconde main, n'est jamais tout à fait neutre. Porteur d'une histoire (celle qui l'a mis en contact avec un humain) dont certains éléments de son aspect général dévoilent les traces, il est nécessairement évocateur d'une mémoire qui, si elle est souvent anonyme et exogène au geste théâtral, vient prendre une part active à l'écriture scénique. Cette dose de mémoire inconnue, étrangère, s'impose comme une aide supplémentaire au déplacement d'un usage 
traditionnellement figuratif du costume de scène.

Pour l'acteur, intégrer l'histoire secrète d'un costume, c'est donc accepter l'invitation au voyage qu'il propose. Selon la belle expression de Laurence Chable en effet, le costume est bien plus qu'un vêtement, il est un «embarquement ${ }^{48}$ ", terme qui laisse à la fois entendre l'idée d'un chargement (ludique, même s'il peut contraindre physiquement l'acteur, l'empêcher) comme celle d'un déplacement (celui qui oriente l'acteur dans le travail qui consiste à se " débarrasser d'un soi-même ${ }^{49}$ " encombrant). Par ailleurs, en acceptant de se laisser ainsi déplacer par le textile, l'interprète acte intimement l'autonomie signifiante de la matière textile ${ }^{50}$ et plus encore la nécessité profonde d'entrer en relation avec elle et ce qu'elle transporte,

parce que ces éléments font déjà la moitié du chemin pour toi et avec toi. Ce sont des intensités à part entière. Ça transporte, ça transfère, ça crible (au sens de tri). Le costume n'est pas là pour faire accord avec toi, il a son autonomie, il crée une sorte de hors-champ de toi, très précieux. C'est aussi une instance. [...] Un costume, c'est une vibration, c'est une trace que tu vas pouvoir suivre, écouter ${ }^{51}$.

Ce jeu de piste est particulièrement perceptible dans le travail par l'usage de nombreuses pièces de vêtement tirées de costumes du passé (fraise, jupon, chapeau, perruque, fragment d'armure...) venant prendre place - de façon quasi permanente dans des assemblages hétérogènes d'éléments surajoutés à une base de costume moderne ou contemporain - principe d'empilement et de combinaison des vêtements qui semble être à la base de la conception du costume chez François Tanguy. En un passage, ces éléments de costume font alors effectivement vibrer l'espace et les corps, c'est-à-dire qu'ils créent de la résonnance dans la lecture entre ce qui se présente concrètement au plateau (les matières et les corps) et ces matières-mémoires transportées.

Figure 8 : Ricercar (2007). Frode Bjørnstad, Claudie Douet, Laurence Chable, Boris Sirdey

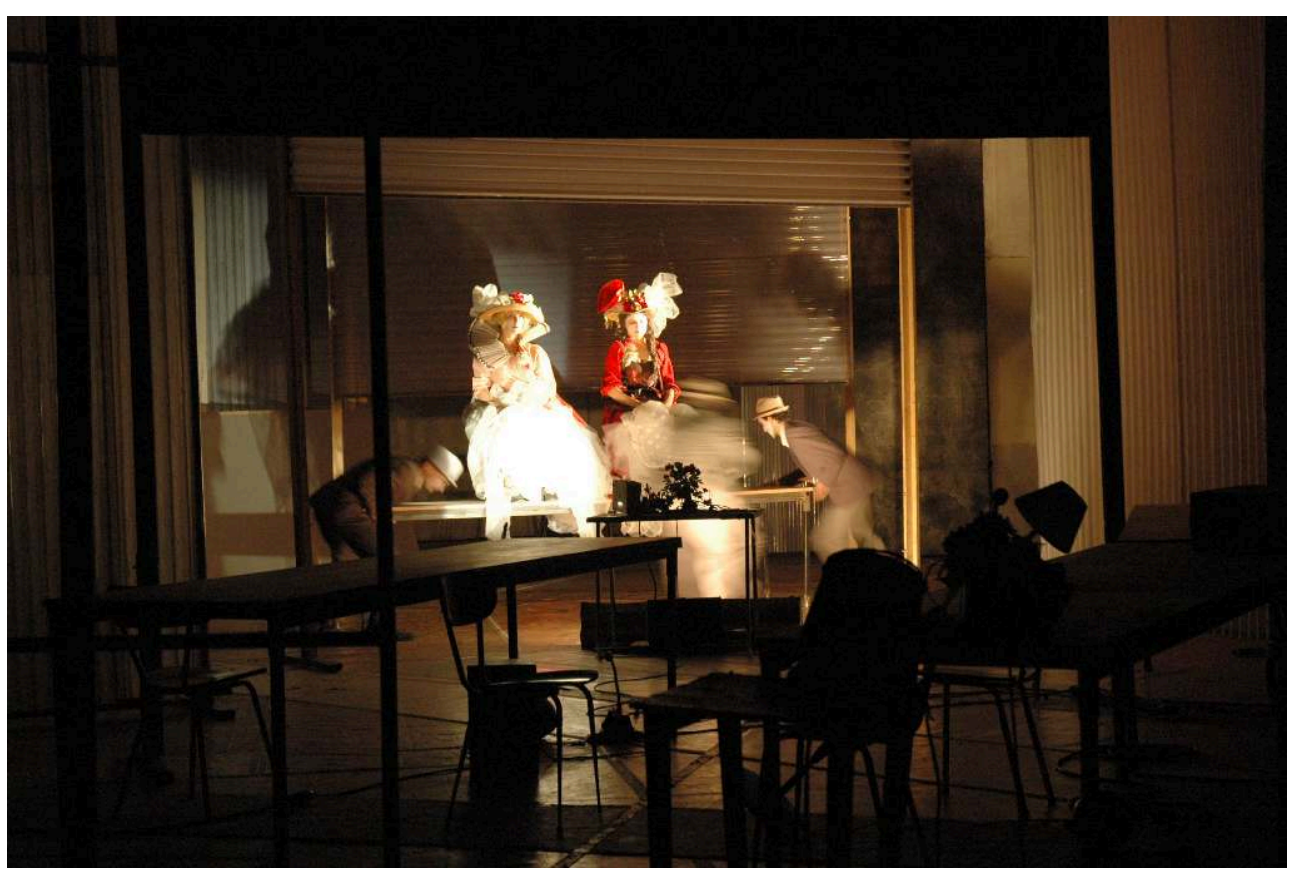

(Crédit photo : Didier Grappe) 


\section{aussi importants. Parallèlement à la dimension historique du costume, il y a en effet} l'histoire propre du costume.

Cette mémoire est d'abord inhérente à n'importe quel tissu de récupération. Dans un habit qui a déjà été porté semble en effet s'inscrire une empreinte fantomatique, et à travers elle la mémoire invisible et muette d'un corps dont n'apparaissent plus que quelques traces en suspension dans l'air environnant: une taille, une carrure, parfois un âge et plus rarement encore le souvenir d'événements particuliers (qui ne manquent pas d'apparaître néanmoins dans les créations du Radeau, où par exemple les robes de mariées, complètes ou réduites à un seul jupon, sont très souvent employées). Mêlée à la masse des vêtements qui emplissent les rayonnages de vide-greniers et des Emmaüs, cette mémoire-textile perd toute traçabilité en se fondant dans le maelström anonyme d'anciennes garde-robes éventrées et dispersées. Mais même réduite au silence, il n'en reste pas moins que cette mémoire se devine. Entrer dans un vêtement de récupération, c'est donc s'introduire dans l'empreinte d'un autre corps, et ce faisant dans la mémoire de ce corps autre.

Si cette mémoire de récupération s'incarne dans tout vêtement de seconde main, il semble d'autre part qu'elle se révèle de manière privilégiée par les stigmates d'usage et d'usure qui modifient l'état général du textile. Contre une conception ornementale et décorative qui cache la moindre altération, se dessine alors une conception du costume pauvre. Comme l'affirme en effet Laurence Chable, «si c'est un peu déchiré, un peu crassepouille, il y a déjà une histoire... que tu ne connais $\operatorname{pas}^{52} »$ et qui, là encore, aide à trouver l'ailleurs nécessaire au décentrement de l'acteur. Tous les signes d'usure (trous, effilochage, couleurs délavées...) participent donc de cette présence manifeste de la mémoire car ils sont les témoignages sensibles d'une érosion de l'étoffe à l'épreuve du temps. Plutôt que d'être masquées, ces blessures sont assumées voire recherchées par François Tanguy. Chacun de ces signes rend compte d'un accident, d'une mésaventure de la matière, qui peut être totalement extérieure à la création (dans ce cas le vêtement a été choisi avec ou pour son usure) comme elle peut être la trace d'un accident de répétition (c'est le travail lui-même qui peut avoir endommagé le costume sans avoir pour autant entamé la nécessité de son emploi ; au contraire, cette usure s'intègre parfaitement à l'histoire du vêtement dans le processus artistique). En réponse à ces accidents se multiplient diverses stratégies: bricolages, réparations, rafistolages précaires sont légion et, en la matière, la discrétion est toute relative. Dans cette intention en effet, comme en atteste le vieux chapeau de paille que Fosco Corliano porte dans la première séquence de Onzième, l'outil de prédilection est moins l'aiguille et le fil que le chatterton orange - signe que ces cicatrisations ostensibles s'intègrent historiquement au processus de création en devenant les marqueurs flagrants d'une inscription temporelle sur le costume et (à la manière du Kintsugi ${ }^{53}$ japonais) d'une exaltation de ces meurtrissures subies par la matière.

Puisqu'ils sont usables et réparables jusqu'à l'épuisement, certains de ces costumes, à l'instar des éléments scénographiques dont nous traitions précédemment, se doublent enfin d'une mémoire secondaire, qui enregistre les différentes étapes du parcours artistique du vêtement au sein même de la compagnie. Cette autre occurrence d'une rémanence matérielle redouble la nature "d'embarquement » du costume dont parlait Laurence Chable. En plus de toutes les caractéristiques mnésiques que nous venons d'analyser, c'est le corps fantôme des interprètes qui revêtirent ce costume dans le 
passé de la compagnie qui est convoqué. À ce titre, un exemple une fois encore tiré de Onzième est tout à fait parlant : le manteau du capitaine Lebiadkine. Dans les dernières minutes du spectacle, Vincent Joly porte la parole du personnage extrait des Démons de Dostoïevski. La figure qu'il compose à partir de ce texte tient en grande partie à la grosse moustache postiche et surtout à l'allure du long manteau de laine bleue au large col de fourrure qu'il porte fermé. Légèrement trop grand pour lui, il crée sur lui le corps d'une figure dégingandée dont la démarche et la parole sont pareillement maladroites. Si cette silhouette rappelle celle tout aussi frêle et sautillante que celle de Frode Bjønrstad (un des acteurs piliers du Radeau), c'est très certainement par la puissance d'évocation de ce long manteau bleu que l'acteur norvégien portait lui-même dans une séquence centrale de Ricercar (2007). D'une création à l'autre, d'un corps à l'autre, c'est une figure assez familière qui apparaît et qui, comme un personnage de la Commedia dell'arte, transite d'un spectacle à l'autre, d'un acteur à un autre en toute autonomie. Mais plus encore, c'est le souvenir de ce corps fantôme qui se meut dans le long manteau à col de fourrure qui revient. Geste suprême et radical de retrait de l'acteur au profit du souvenir d'un autre acteur, le remploi de ce costume œuvre comme si la présence de Frode Bjørnstad perdurait malgré son absence au plateau ${ }^{54}$.

Figure 9 : Soubresaut (2016). Vincent Joly, Muriel Hélary, Frode Bjørnstad, Didier Bardoux, Laurence Chable, Karine Pierre, Ida Hertu

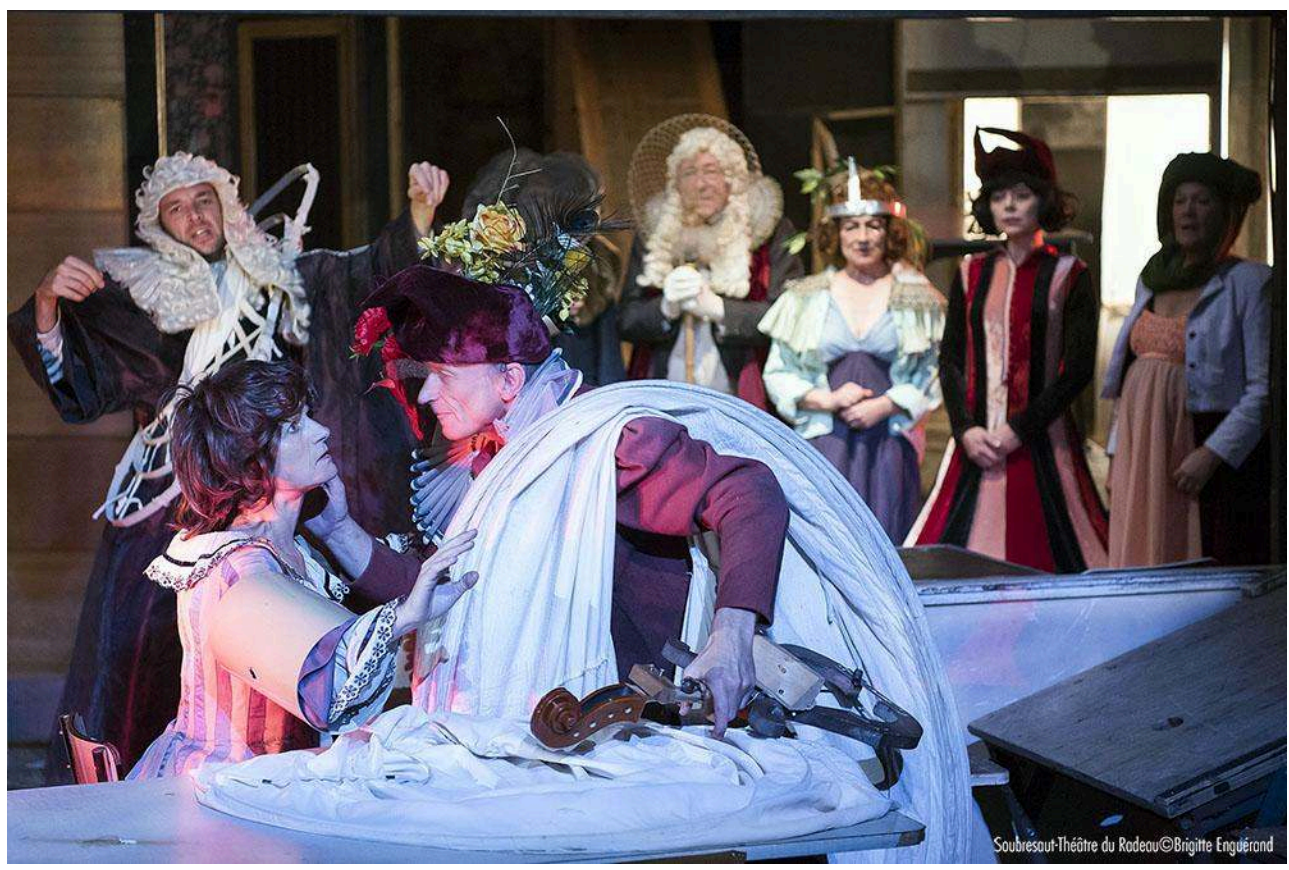

(Crédit photo : Brigitte Enguérand)

Qu'elles entrent dans la composition des éléments scénographiques ou des costumes, les matières, dans les espaces du Radeau, n'ont donc pas vocation à jouer les utilités. La mise en présence au plateau de leur vitalité suscite l'attention esthétique et fait spectacle, elle requiert le jeu théâtral, plus qu'elle n'est requise et chargée d'intentions à des fins de signification ou de narration. Pour l'acteur, le jeu consiste donc moins à mettre en mouvement son corps et sa voix dans un espace dont la composition importe peu qu'à créer du liant entre sa personne et les matières alentours; il intercède dans l'élaboration d'une forme de milieu au sein duquel corps, choses et matières œuvrent de 
concert - un théâtre concret ou, selon le vocabulaire mésologique formulé par Augustin Berque, un théâtre de la "concrescence » (étymologiquement, "qui croît ensemble ») et de la « co-suscitation » dans lequel

les choses du milieu ne sont pas des objets substantiels, subsistant dans leur en-soi ; elles sont toujours en train de se faire dans leur interaction avec le sujet. Réciproquement, le sujet aussi toujours en train de se faire dans son interaction avec les choses ; autrement dit, dans son moment structurel avec le milieu ${ }^{55.56}$

\section{BIBLIOGRAPHIE}

BENNETT, Jane, Vibrant matter. A political ecology of things, Durham/London, Duke University Press, 2010.

BERQUE, Augustin, La Mésologie, pourquoi et pour quoi faire?, Nanterre, Presses Universitaires de Paris Ouest, coll. Essais et conférences, 2014.

CAMBRON, Maxence, « De la remembrance théâtrale : poétique et politique de la mémoire dans la création scénique contemporaine en Europe (François Tanguy, Christoph Marthaler, Maguy Marin), Thèse de doctorat, sous la direction de M. le professeur Amos Fergombé, Département Arts du spectacle, Université d'Artois, 2016.

CHARLET, Emilie, COULON, Aurélie, NOEL, Anne-Sophie (dir.), « L'objet », Revue Agôn [en ligne], Dossier $n^{\circ} 4,2011$.

DAGOGNET, François, Rematérialiser. Matières et matérialismes, Paris, Vrin, 1985.

INGOLD, Tim, Faire. Anthropologie, archéologie, art et architecture, trad. Hervé Gosselin et HichamStéphane Afeissa, Bellevaux, Editions Dehors, 2017.

KANTOR, Tadeusz, Le Théâtre de la mort. [1977] Textes réunis et présentés par Denis Bablet, Lausanne, L'Age d'Homme, coll. Théâtre XX ${ }^{\mathrm{e}}$ siècle, 2004.

MANGANARO, Jean-Paul, François Tanguy et le Radeau, Paris, P.O.L., 2008.

MATTEOLI, Jean-Luc, L'Objet pauvre : mémoire et quotidien sur les scènes contemporaines françaises, Rennes, Presses Universitaires de Rennes, coll. Le Spectaculaire. Série Théâtre, 2011.

De MÈREDIEU, Florence, Histoire matérielle et immatérielle de l'art moderne et contemporain [1994], 3

ème éd., Paris, Larousse, coll. In extenso, 2011.

OLIVIER, Laurent, Le Sombre abîme du temps. Mémoire et archéologie, Paris, Seuil, coll. La Couleur des idées, 2008.

TANGUY, François, « Le théâtre comme œuvre plastique », entretien avec Jean-Pierre Berthier, in Jean-Pierre Berthier (dir.), Des images et du théâtre : dossier pédagogique, Paris, CNDP, 1998.

TACKELS, Bruno, François Tanguy et le Théâtre du Radeau. Écrivains de plateau 2, Besançon, Les Solitaires intempestifs, coll. Du Désavantage du vent, 2005.

VAUTRIN, Éric (dir.), « Variations Radeau », Théâtre/Public, n² 214, Montreuil, Editions Théâtrales, oct-déc. 2014.

\section{NOTES}

1. Le Théâtre du Radeau a été fondé au Mans à la fin des années 1970 à l'initiative de l'actrice Laurence Chable. Rejoint par François Tanguy en 1982, la compagnie a depuis lors proposé dix- 
sept créations, internationalement reconnues. Depuis le début des années 1990, le Radeau occupe et gère La Fonderie, siège de la compagnie devenu, au fil des années, lieu d'accueil pour d'autres équipes artistiques en création, mais aussi lieu de militance et de réflexion citoyenne.

2. Pour n'évoquer ici que la dimension proprement matérielle de son théâtre, à laquelle pourrait également s'adjoindre la matérialité élargie des sons, des voix, des langues et langages poétiques qui participent tout autant de son écriture scénique.

3. François Tanguy, cité par Daniel Loayza dans le dossier de presse de coda pour le Théâtre de l'Odéon [En ligne] URL: https://www.theatre-odeon.eu/media/odeon/file_31_dpd_5_03.pdf [Consulté le 08/01/2020].

4. Courant philosophique apparu à la fin du XXe siècle ayant entrepris de repenser les rapports entre l'Homme et les choses, l'Homme et la nature, les nouveaux matérialismes (nous employons l'expression au pluriel pour éviter de la figer en système fermé, ce qui n'est guère le cas) s'appuient notamment sur l'effacement de la dualité moderne séparant l'humain du non-humain et défendent au contraire un rééquilibrage de ces rapports, pour les envisager dans une perspective plus équitable voire interactive.

5. Ce qui, du point de vue des normes sécuritaires, n'est pas sans poser problème à la compagnie, qui doit aussi intégrer à son dispositif technique la prise en compte des nombreuses règles de sécurité qui régissent l'implantation des scénographiques dans les théâtres français et européens. Comme nous l'expliquait François Fauvel (directeur technique de la compagnie), plusieurs de ces matières (preuve de leur inadéquation de fond avec le théâtre d'aujourd'hui), non-ignifugées, quand elles ne sont pas rejetées pour ces aspects sécuritaires, doivent faire l'objet de traitements spécifiques par la compagnie. D'autres solutions, avec une incidence directe sur le projet esthétique, sont par ailleurs mises en œuvre comme par exemple le positionnement de certaines matières à plus de deux mètres d'autres dont l'inflammabilité serait rapidement contagieuse, ou des sources d'éclairage qui (sans compter celles qui, là encore, n'appartiennent pas au champ traditionnel du théâtre, comme d'anciens projecteurs à diapositives) sont dissimulées directement dans l'espace scénique.

6. De MÈREDIEU, Florence, Histoire matérielle et immatérielle de l'art moderne et contemporain [1994],

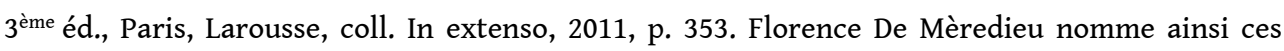
matériaux pour les opposer aux matériaux nobles qui servent traditionnellement de matière première à l'art, mais également pour désigner ces matières artificielles issues de procédés industriels dont certaines ne sont que de peu de valeur.

7. Ibid., pp. 346-367.

8. Tadeusz Kantor, Le Théâtre de la mort. [1977] Textes réunis et présentés par Denis Bablet, Lausanne, L'Âge d'Homme, coll. Théâtre XX'e siècle, 2004, p. 214.

9. Ibid., p. 71.

10. Ibid., p. 257.

11. Ibid., p. 32.

12. Ces principes esthétiques permettent en effet une baisse des coûts de fourniture et de construction de la scénographie, et présentent également des atouts écologiques (la récupération de matériaux voués à la déchetterie permet leur revalorisation et ainsi une limitation des conséquences polluantes de leur traitement).

13. Jean-Luc Mattéoli, L'Objet pauvre : mémoire et quotidien sur les scènes contemporaines françaises, Rennes, Presses Universitaires de Rennes, coll. Le Spectaculaire, Série Théâtre, 2011, p. 63.

14. Antoine Compagnon, Les Chiffonniers de Paris, Paris, Gallimard, coll. Bibliothèque illustrée des histoires, 2017.

15. Scénographe attitré de la compagnie depuis son arrivée, François Tanguy - selon les bibles des spectacles disponibles sur le site du Théâtre du Radeau-partage en revanche les élaborations sonores et lumières avec deux collaborateurs de longue date : Éric Goudard pour le son, François Fauvel pour la lumière (François Fauvel est par ailleurs directeur technique de la 
compagnie). Pour la construction de la scénographie, l'atelier est ouvert à tous, techniciens, acteurs ou non.

16. Pour davantage de détails sur le processus de création au Théâtre du Radeau, voir Maxence Cambron, "La répétition invisible : le travail au Théâtre du Radeau », communication dans le cadre du Colloque International Les Processus de création au théâtre (organisé par Sophie Proust - APC/CEAC/Action Culture, Lille 3, en partenariat avec la rose des vents, le Prato et le Théâtre du Nord - du 16 au 18 avril 2014) (vidéo en ligne : https://live3.univ-lille3.fr/video-recherche/17maxence-cambron-lille-3-la-repetition-invisible-le-travail-au-theatre-du-radeau.html), Marc Bodnar, «Un Radeau ça va lentement » (in BANU, Georges (dir.), Les répétitions. De Stanislavski à aujourd'hui, Arles, Actes Sud, coll. Le Temps du théâtre, 2005, pp. 419-424) et Serge Nail, « Le geste créateur de l'acteur chez François Tanguy (Théâtre du Radeau). Ou quand le jeu se rapproche de «la pure activité de tisserand» [en ligne] (URL : https://journals.openedition.org/doublejeu/ 2268\#bodyftn3)

17. INGOLD, Tim, Faire. Anthropologie, archéologie, art et architecture [2013], trad. Hervé Gosselin et Hicham-Stéphane Afeissa, Bellevaux, Editions Dehors, 2017.

18. Sur cette question, voir ABRAM, David, Comment la terre s'est tue. Pour une écologie des sens, trad. Didier Demorcy et Isabelle Stengers, Paris, La Découverte, coll. Les empêcheurs de penser en rond, 2013.

19. Pour cette citation, et les suivantes, nous nous référons à l'extrait de l'ouvrage de Tim Ingold («Les matériaux de la vie») disponible en ligne dans la traduction de Christel Paris. URL: https://journals.openedition.org/socio-anthropologie/2519.

20. Pour les prochaines citations, KANTOR, Tadeusz, op. cit., pp. 213-214.

21. BENNETT, Jane, Vibrant matter. A political ecology of things, Durham/London, Duke University Press, 2010.

22. Ibid., p. XVIII.

23. MERLEAU-PONTY, Maurice, Phénoménologie de la perception [1945], Paris, Gallimard, coll. TEL, 1976.

24. SPINOZA, L'Ethique, 1677.

25. LATOUR, Bruno, Reassembling the social: An introduction to social life, Oxford, Oxford University Press, 2005.

26. DELEUZE, Gilles, GUATTARI, Félix, Capitalisme et schizophrénie 2 : Mille plateaux, Paris, Éditions de Minuit, coll. Critique, 1980.

27. BENNETT, Jane, op. cit., p. XVIII. Nous traduisons.

28. KANTOR, Tadeusz, op. cit., p. 214.

29. BENNETT, Jane, op. cit., p. XVIII. Nous traduisons.

30. Ibid., p. 2. Nous traduisons.

31. Cf. BERQUE, Augustin, La Mésologie, pourquoi et pour quoi faire?, Nanterre, Presses Universitaires de Paris Ouest, coll. Essais et conférences, 2014.

32. Cf. BERQUE, Augustin, La Pensée paysagère? [2008], Bastia, Editions Eoliennes, 2016.

33. Telle qu'elle est développée par Augustin Berque, la notion de milieu hérite des écrits du philosophe japonais Watsuji Tetsurô (dont Augustin Berque a assuré en 2011 la traduction du livre-somme Fûdo, le milieu humain - Paris, CNRS Editions) et du biologiste allemand Jakob von Uexküll (particulièrement pour l'ouvrage Milieu animal et milieu humain, trad. Charles MartinFréville, Paris, Rivages, 2010).

34. Ce qui peut expliquer la durée des processus de création du Radeau (les répétitions durent en moyenne quatre mois - durée exceptionnelle) et peut également aider à comprendre la chorégraphie permanente des choses dans la scénographie qui, à la manière d'un Giacometti surimprimant les traits dans son dessin ou travaillant le même morceau de plâtre, rend compte de l'insaisissabilité du mouvement par le mouvement même. 
35. Cf. François Tanguy, "Le théâtre comme œuvre plastique ", entretien avec Jean-Pierre Berthier, in BERTHIER, Jean-Pierre (dir.), Des images et du théâtre : dossier pédagogique, Paris, CNDP, 1998.

36. François Dagognet, "Postface» in MONNOYEUR, Françoise, Qu'est-ce que la matière? Regards scientifiques et philosophiques, Paris, Le Livre de Poche, coll. Biblio essais, 2017, p. 145.

37. De MÈREDIEU, Florence, op. cit., p. 349.

38. Sur cette question, envisagée selon une perspective sociologique et philosophique, nous renvoyons le lecteur à l'article de Baptiste Monsaingeon, « De la présence aux restes. Les ombres fantomatiques d'un monde d'objets » [en ligne]. URL : https://journals.openedition.org/socioanthropologie/2443\#authors [consulté le 08/01/2020].

39. Idiolecte propre à la langue poétique (et parfois énigmatique) de François Tanguy, la première occurrence date du texte de présentation des Cantates (2001) dans lequel il écrit, sans autre forme de développement (François Tanguy est plutôt rétif aux explications) : «Aller à vue, dans l'air où les ondes portent les matières, se muent en formes dans le regard, en voix dans l'espace, ou quelque autre perception, selon les vitesses, les résonnances, les traits, les facultés de l'instant. Appeler ces mouvements : des mémorants ». En d'autres termes, les mémorants sont des seuils, des interface entre tout ce qui peut être séparé ou mis en écart.

40. En 2016, une partie des agrès du Radeau ont été prêtés à la Compagnie d'Ores et déjà pour constituer la scénographie de Angelus Novus, Antifaust, création du metteur en scène Sylvain Creuzevault très lié à François Tanguy. Sortis du contexte théâtral qui les voit évoluer ordinairement, ces éléments, dans un spectacle non mis en scène par François Tanguy, se voyaient ainsi chargés d'une nouvelle strate mémorielle.

41. Klaus Michael Grüber (1941-2008), figure importante du renouvellement de la mise en scène allemande à la charnière des années 1970 et 1980 fut un complice très proche de François Tanguy.

42. Cf. HALL, Edward T., La Danse de la vie. Temps culturel, temps vécu, Paris, Seuil, coll. Points essais, 1992, trad. Anne-Lise Hacker.

43. BENNETT, Jane, «Matérialismes métalliques » [en ligne]. URL: https://www.cairn.info/ revue-rue-descartes-2008-1-page-57.htm\#pa8 [consulté le 08/01/2020].

44. François Tanguy, « Le théâtre comme œuvre plastique », art. cit., p. 70.

45. Laurence Chable, « Jeu : et prolongation », propos recueillis par Eric Vautrin, in Eric Vautrin (dir.), "Variations Radeau », Théâtre/Public, n²14, Montreuil, Editions Théâtrales, oct.-déc. 2014, p. 96-115, [en ligne] URL : http://recherchesradeau.org/tp214/lc [consulté le 08/01/2020],

p. 4.

46. Idem, p. 3.

47. Eric Vautrin (dir.), « Variations Radeau », op. cit., pp. 116-117.

48. Idem, p. 6.

49. Idem.

50. Ce qui n'est pas sans rappeler ce que Roland Barthes réclamait dans son fameux article de 1955 sur «Les Maladies du costume de théâtre " : "le bon costume de théâtre doit être assez matériel pour signifier et assez transparent pour ne pas constituer ses signes en parasites ». Cf. Roland Barthes, "Les maladies du costume de théâtre ", in Roland Barthes, Écrits sur le théâtre, Paris, Le Seuil, Point Essais, pp. 137-146.

51. Laurence Chable, art. cit., p. 6.

52. Ibid.

53. Technique traditionnelle japonaise, le Kintsugi consiste à réparer les vases, assiettes et autres porcelaines et céramiques brisées non en masquant les jointures de l'objet, mais au contraire en les mettant en valeur par l'ajout d'une poudre d'or au mélange faisant office de colle. 
54. Ajoutons que certains des costumes de la compagnie ont également été récupérés à la suite de dons d'autres compagnies ou metteurs en scène (comme Matthias Langhoff), ce qui ne fait que renforcer le caractère mémoriel de ces costumes, cette fois dans une perspective métathéâtrale.

55. BERQUE, Augustin, op. cit., p. 55.

56. L'auteur tient ici à remercier très chaleureusement les coordinatrices de ce dossier pour leur conseils avisés et bienveillants qui ont été d'une aide précieuse dans le développement de cette étude.

\section{RÉSUMÉS}

L'article analyse le travail scénographique de François Tanguy en se concentrant sur le statut particulier qu'il confère aux matières, tant pour leur participation à la conception visuelle du spectacle que pour leur fonction dramaturgique singulière.

Inscrite dans le cadre d'une esthétique de la récupération, cette relation aux matières pauvres se caractérise d'abord par le souci de libérer ces dernières de toutes fonctions utilitaires, illustratives ou figuratives afin de mettre en branle leurs vibrations propres et permettre de les envisager tels des partenaires de jeu pour les acteurs et actrices du Théâtre du Radeau. S'intéressant également à leur "vécu ", l'art de François Tanguy semble par ailleurs considérer ces matières comme des surfaces mémorielles dont le statut de « reste », au sens archéologique du terme, participe pleinement de l'œuvre.

Postulant une contiguïté philosophique entre cette esthétique de la matière et les thèses des nouveaux matérialismes, l'étude propose une lecture de l'œuvre de François Tanguy à l'aune de ce courant de pensée.

\section{INDEX}

Mots-clés : agentivité, costume, nouveaux matérialismes, matière, mémoire, récupération, scénographie, Tanguy (François), Théâtre du Radeau

\section{AUTEUR}

\section{MAXENCE CAMBRON}

Maxence Cambron est docteur en arts du spectacle. Il est contractuel d'enseignement en Arts de la scène à l'Université de Lille ainsi que chargé de cours en Arts du spectacle aux Universités d'Artois et de Strasbourg.

Ses travaux de recherche au sein du Centre de recherche Textes et cultures (EA 4028, Université d'Artois) portent sur la création théâtrale contemporaine (scènes et dramaturgies), l'histoire des politiques culturelles en matière de théâtre en France et sur les pratiques théâtrales amateurs. Il est l'auteur d'une thèse réalisée sous la direction du Pr Amos Fergombé et soutenue en 2016 intitulée De la remembrance théâtrale : poétique et politique de la mémoire dans la création scénique contemporaine en Europe (François Tanguy, Christoph Marthaler, Maguy Marin).

maxence.cambron@orange.fr 